\title{
An Ultrasensitive CMOS Magnetic Biosensor Array with Correlated Double Counting Noise Suppression
}

\author{
Hua Wang, Shohei Kosai, Constantine Sideris, and Ali Hajimiri \\ California Institute of Technology, Pasadena, California, 91125, USA
}

\begin{abstract}
This paper presents a scalable and ultrasensitive frequency-shift magnetic biosensing array scheme. The theoretical limit of the sensor noise floor is shown to be dominated by the phase noise of the sensing oscillators. To increase the sensitivity, a noise suppression technique, Correlated Double Counting (CDC), is proposed with no power overhead. As an implementation example, a 64-cell sensor array is designed in a standard $65 \mathrm{~nm}$ CMOS process. The CDC scheme achieves an additional 6dB noise suppression. The magnetic sensing capability of the presented sensor is verified by detecting micron size magnetic particles with an SNR of 14.6dB for a single bead and an effective dynamic range of at least $74.5 \mathrm{~dB}$.
\end{abstract}

Index Terms - Biomedical transducers, biomagnetics, biomedical measurements, medical diagnosis, CMOS integrated circuits, integrated circuit noise.

\section{INTRODUCTION}

Portable microarrays are promising for Point-of-Care (POC) medical applications, such as disease detection, control, and monitoring, where the key technical challenges are hand-held portability, high sensitivity, battery-level power consumption, and low cost.

Magnetic biosensors in the form of portable microarrays are proposed to replace or augment conventional fluorescent sensors, which need bulky and expensive optical systems. However, all magnetic sensors reported thus far require external bias magnetic fields and/or complicated postprocessing, limiting their form factor and cost [1-3]. An ultrasensitive frequency-shift sensing scheme has been thus proposed to address these issues [4]. In this paper, the fundamental noise floor of this sensing scheme is modeled, and a noise suppression technique, Correlated Double Counting (CDC), is proposed and implemented which achieves $6 \mathrm{~dB}$ of noise reduction without any power overhead in the sensor array presented in this paper.

This paper is organized as follows: Section II introduces the frequency-shift magnetic sensing scheme and proposes the noise suppression technique. Section III presents a CMOS sensor array implementing the technique. The sensor's noise suppression performance is characterized in section IV and its magnetic sensing capability is verified in section $\mathrm{V}$.

\section{SENSING SCHEME AND NOISE SUPPRESSION TECHNIQUE}

Magnetic sensing is typically accomplished by a sandwich bioassay. The target molecules are first captured by predeposited molecular probes onto the sensor surface. Magnetic particles (labels) coated with other types of probes are then added and immobilized by the captured target molecules. Therefore, one can detect the presence of target molecules in the sample by sensing the magnetic labels left on the surface.

\section{A. Frequency Shift Magnetic Sensing and Noise Analysis}

In the frequency-shift sensing scheme, the inductor of an on-chip LC oscillator functions as the sensing core. The AC current through the inductor generates a magnetic field to polarize the present magnetic particles. This increases the total magnetic energy in the space and thus leads to an effective increase in the sensing inductor inductance. Consequently, the on-chip LC oscillator senses this inductance increase via a corresponding downshift in oscillation frequency [4].

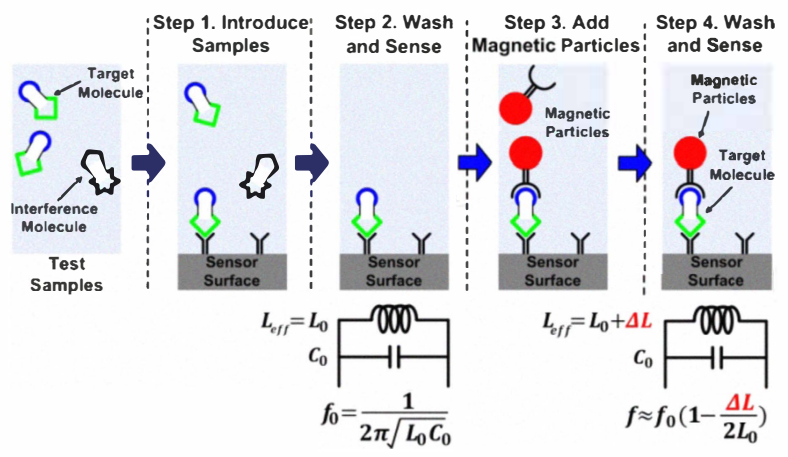

Fig. 1. Inductive frequency shift magnetic sensing scheme

The oscillation frequency is measured by frequency counting (i.e. recording the number of rising edges within a given counting time $T$ ). Therefore, the noise floor is set by the accumulated jitter $\sigma_{T}^{2}$ of the waveform which is determined by its phase noise spectrum, $S_{\phi}(\omega)$. The relative frequency error $\sigma_{\Delta f / f_{0}}^{2}$ in frequency counting can thus be calculated as

$$
\begin{aligned}
\sigma_{\Delta f / f_{0}}^{2} & =\frac{(\Delta f)^{2}}{f_{0}^{2}}=\frac{\sigma_{T}^{2}}{T^{2}}=\frac{1}{T^{2} \omega_{0}^{2}} E\left\{[\phi(T)-\phi(0)]^{2}\right\} \\
& =\frac{4}{\pi \omega_{0}^{2} T^{2}} \int_{0}^{+\infty} S_{\phi}(\omega) \sin ^{2}\left[\frac{\omega T}{2}\right] d \omega,
\end{aligned}
$$

where $f_{0}=\omega_{0} / 2 \pi$ is the oscillation frequency and $\omega$ is the offset frequency for the SSB phase noise $S_{\phi}(\omega)[5]$. $(\Delta f)^{2}$ represents the frequency uncertainty during counting.

At a short counting time $\left(T \ll 2 \pi / \omega_{1 / f^{3}}\right.$, where $\omega_{1 / f^{3}}$ is the $1 / f^{3}$ corner frequency of the phase noise $\left.S_{\phi}(\omega)\right), 1 / f^{2}$ noise dominates the jitter and $\sigma_{\Delta f / f_{0}}^{2}$ is inversely proportional to $T$ : 


$$
\sigma_{\Delta f / f_{0}}^{2}=\frac{\sigma_{T}^{2}}{T^{2}}=\frac{\kappa^{2} T}{T^{2}}=\frac{\kappa^{2}}{T},
$$

where $\kappa$ is the $1 / f^{2}$ jitter coefficient for the oscillator. For a large counting interval $\left(T \gg 2 \pi / \omega_{1 / f^{3}}\right), 1 / f^{3}$ phase noise dominates and leads to $\sigma_{\Delta f / f_{0}}^{2}$ independent of $T$ :

$$
\sigma_{\Delta f / f_{0}}^{2}=\frac{\sigma_{T}^{2}}{T^{2}}=\frac{\zeta^{2} T^{2}}{T^{2}}=\zeta^{2},
$$

where $\zeta$ is the $1 / f^{3}$ jitter coefficient [5]. Moreover, due to the uncertainty principle (effect of finite counting window length), there exists a measurement error proportional to $1 / f_{0}^{2} T^{2}$. The total frequency uncertainty can be plotted, which shows that $\zeta^{2}$ determines the ultimate sensor noise floor (Fig.3).

In practical sensor designs, differential sensing is used by pairing a sensing oscillator with a reference oscillator [4]. Their frequency difference is taken as the sensor output to eliminate common-mode drift due to effects, such as supply and temperature variations. In differential sensing, an extra factor of 2 appears in $\sigma_{\Delta f / f_{0}}^{2}$ for noise power doubling,

$$
\sigma_{\Delta f / f_{0}}^{2}=\frac{2 \times 4}{\pi \omega_{0}^{2} T^{2}} \int_{0}^{+\infty} S_{\phi}(\omega) \sin ^{2}\left[\frac{\omega T}{2}\right] d \omega .
$$

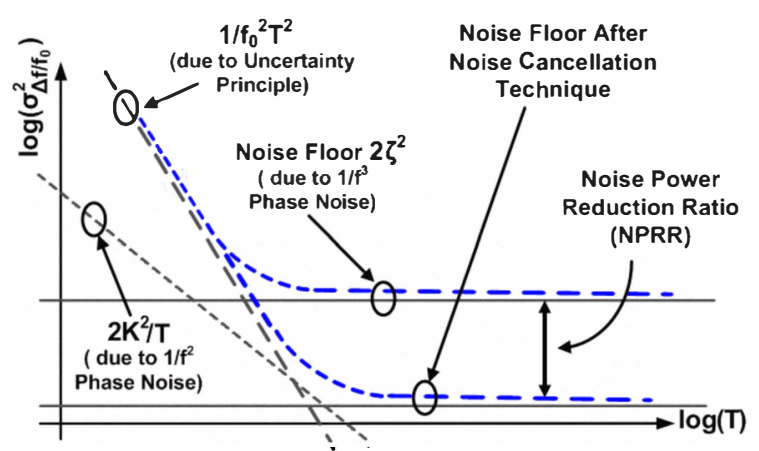

Fig. 3. Noise floor for oscillator based frequency shift sensing.

\section{B. Correlated Double Counting (CDC) Noise Suppression Technique}

Based on the analysis above, $1 / f^{3}$ phase noise (captured by the $\zeta^{2}$ coefficient) sets the minimum sensor noise floor. This $1 / f^{3}$ phase noise is determined by the flicker noise of the devices and the waveform properties of the oscillators and is generally difficult to reduce beyond a certain level. Based on (3), the $1 / f^{3}$ noise jitter $\left(\zeta^{2} T^{2}\right)$ accumulates at a faster rate due to its long correlation time. While this correlation results in a higher integrated noise power, it also creates a possibility of noise cancellation.

In a normal differential sensing, the jitters are uncorrelated between the sensing and the reference oscillator, which gives the extra factor of 2 in (4). However, if the $1 / f^{3}$ phase noise is correlated between the two oscillators, this correlated noise can be subtracted by a correlated double counting (CDC) scheme, where subtracting one frequency count from the other will reduce the correlated noise component and thus lowers the measurement uncertainty floor as shown in Fig. 3. Since
$1 / f^{3}$ phase noise is mainly from flicker noise upconversion of the active devices in a CMOS oscillator, this suggests designing the two oscillators in a way to increase the flicker noise correlation between the reference and sensing cores, e.g. active core sharing, during differential operation (Fig.4).

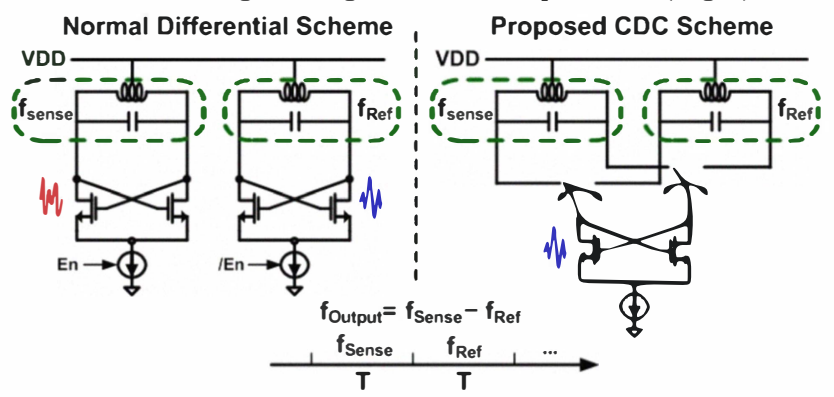

Fig. 4. Normal differential sensing scheme and CDC scheme.

This noise suppression technique is analogous to the Correlated Double Sampling (CDS) often used in image sensors. This proposed CDC scheme for our sensor does not increase the power consumption or chip area. Moreover, this CDC scheme with oscillator based frequency-shift sensing provides a general sensor design methodology which can be applied to any type of sensor that measures LC resonant changes, e.g. capacitive sensing in pressure sensors [6].

The proposed scheme can be modeled mathematically. Assuming $\phi(t)$ as the correlated random phase of the oscillator waveform, the effective noise for CDC scheme is:

$$
\begin{aligned}
\sigma_{\Delta f / f_{0}}^{2}= & \frac{1}{T^{2} \omega_{0}^{2}} E\left\{[\phi(2 T)-\phi(T)-[\phi(T)-\phi(0)]]^{2}\right\} \\
& =\frac{16}{\pi \omega_{0}^{2} T^{2}} \int_{0}^{+\infty} S_{\phi}(\omega) \sin ^{4}\left[\frac{\omega T}{2}\right] d \omega,
\end{aligned}
$$

where $T$ is the counting time and $\omega_{0}$ is the nominal oscillation frequency. From (4) and (5), noise shaping functions for normal differential scheme and CDC scheme can be defined and plotted (Fig. 5). Compared with the normal differential case, the CDC provides a $4^{\text {th }}$ order zero at low frequency offsets enabling a greater suppression on the $1 / f^{3}$ phase noise.

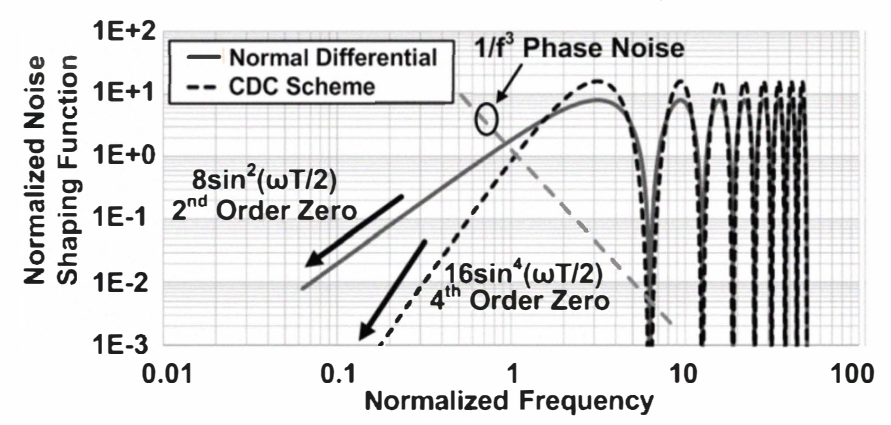

Fig. 5. Noise Shaping Function.

Furthermore, assuming a $1 / f^{3}$ phase noise profile of $S_{\phi, 1 / f^{3}}(\omega)$, the Noise Power Reduction Ratio (NPRR) between the two schemes can be numerically calculated as 


$$
\begin{aligned}
N P R R & =\frac{\sigma_{\Delta f / f_{0, d i f f}^{2}}^{2}}{\sigma_{\Delta f / f_{0, C D F C}}^{2}}=\frac{\frac{8}{\pi \omega_{0}^{2} T^{2}} \int_{0}^{+\infty} S_{\phi, \frac{1}{f^{3}}}(\omega) \sin ^{2}\left[\frac{\omega T}{2}\right] d \omega}{\frac{16}{\pi \omega_{0}^{2} T^{2}} \int_{0}^{+\infty} S_{\phi, \frac{1}{f^{3}}}(\omega) \sin ^{4}\left[\frac{\omega T}{2}\right] d \omega} \\
& =2 \zeta^{2} / \sigma_{\Delta f / f_{0, C D F C}^{2}} \approx 9.6 d B,
\end{aligned}
$$

where calculating $\zeta^{2}$ for a given $1 / f^{3}$ phase noise can be found in [7]. This shows a noise reduction ratio of up to $9.6 \mathrm{~dB}$ can be achieved by the proposed CDC scheme.

\section{SENSOR SYSTEM IMPLEMENTATION}

In this section, a CMOS magnetic sensor array with the proposed CDC scheme is presented as a design example.

\section{A. Quad-Core Sensor Cell}

Each sensor cell in the array is designed with four sensing sites (four sensing inductors) where any of the four can be selected to function as the reference sensor and the other three as active sensors (Fig. 6). To maintain correlation of the $1 / f^{3}$ phase noise between differential sensing, the four sensing inductors are designed to share the same cross-coupled core, whose up-converted flicker noise dominates the $1 / f^{3}$ phase noise. NMOS switches are used to couple the desired LC tank with the active core. The switches are optimized to minimize their $1 / f^{3}$ phase noise which is uncorrelated during differential sensing and cannot be suppressed by the CDC scheme. The sensing oscillators' nominal frequency is $1 \mathrm{GHz}$.

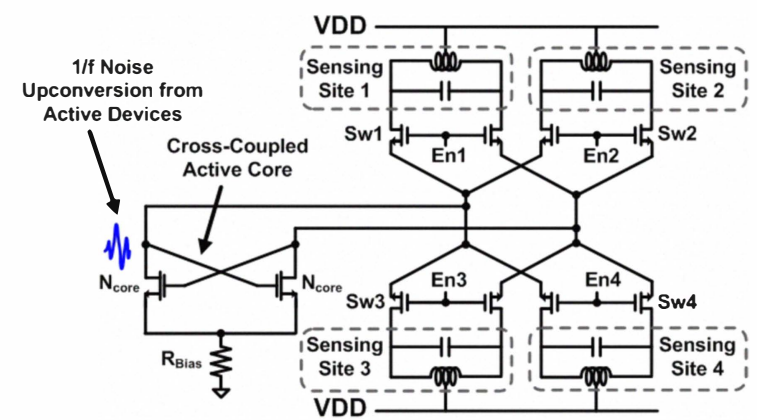

Fig. 6. Schematic for the quad-core CDFC sensor cell.

\section{B. Sensor System Architecture}

The complete sensor system architecture is shown in Fig.7. Sixteen quad-cell sensor cells are implemented on the same CMOS chip for high throughput. Multiplexers are designed to feed the sensing oscillator output to the on-chip frequency counter. Since only one of the sensing sites in each quad-cell is needed to act as the reference, each chip has 48 useful sensor sites overall. Moreover, this architecture is scalable to a large scale array on one chip. In addition, since the sensor chip's IOs only have DC supply and digital inputs/outputs, multiple chips can be easily tiled for ultra-high throughput applications, such as genomic sequencing and genotyping.

The chip microphotograph is shown in Fig.8, with a zoomin view of the quad-core sensor cell. The sensing inductor is a
$120 \mu \mathrm{m}$ 6-turn symmetric spiral with $\mathrm{Q}$ of 6.8 at $1 \mathrm{GHz}$. The sensing inductor spacing of $60 \mu \mathrm{m}$ is chosen to balance the integration level with the coupling from adjacent cells. The total system power consumption is $80 \mathrm{~mW}$.

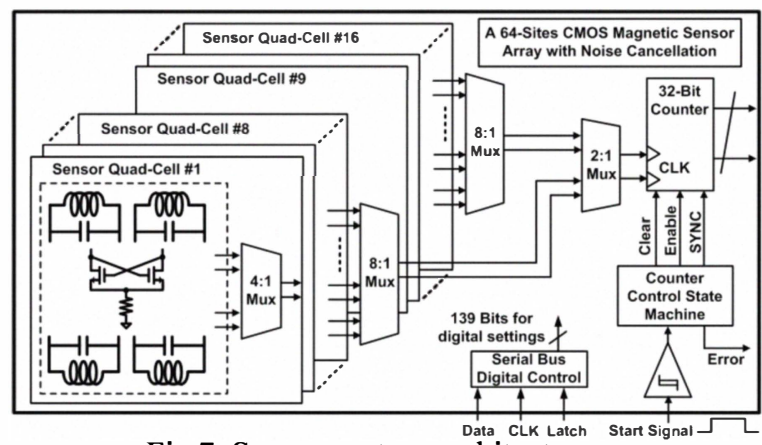

Fig.7. Sensor system architecture.

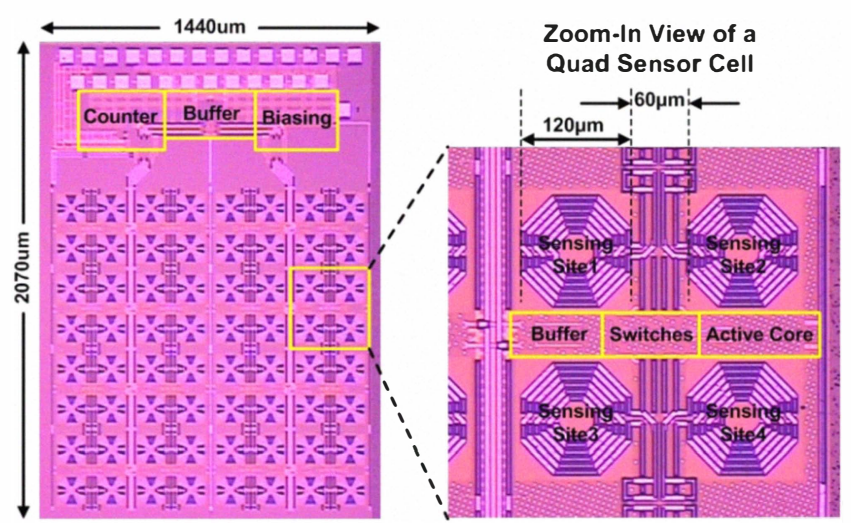

Fig.8. Chip microphotograph.

\section{SENSOR ElECtrical PERformanCE}

The sensor oscillator's phase noise is measured with an RDL phase noise analyzer, achieving $-63.1 \mathrm{dBc} / \mathrm{Hz}$ and $135.3 \mathrm{dBc} / \mathrm{Hz}$ at $1 \mathrm{kHz}$ and $1 \mathrm{MHz}$ offsets, respectively (Fig.9).

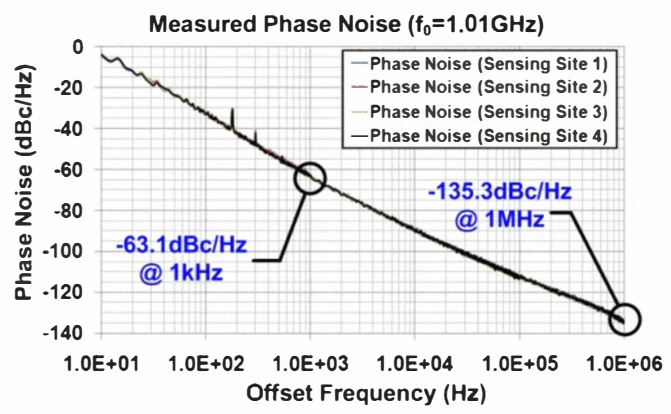

Fig.9. Measured sensor oscillator phase noise.

The noise suppression functionality of the proposed CDC scheme is verified through frequency counting. The standard deviation for relative frequency is measured with respect to different counting time $T$ (Fig.10). The correlated noise case is sampled from one quad-core cell, while the normal differential case is measured using oscillators which are from different but spatially adjacent cores to cancel their thermal drift. The slope 
of $\sigma_{\Delta f / f_{0}}$ (by uncertainty principle) is $-20 \mathrm{~dB} /$ decade, which agrees with the analysis in Section II A.

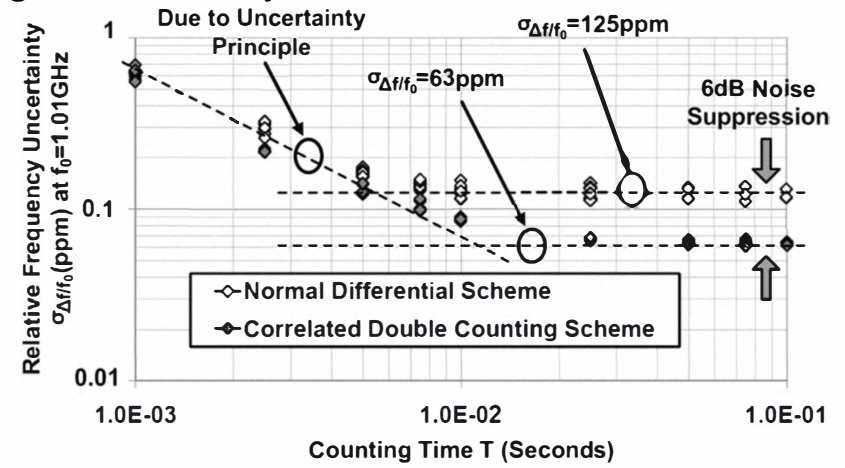

Fig.10. Frequency counting Std. with different counting time.

At large counting intervals $(T>0.01 s)$, the measured sensor noise reaches the minimal noise floor set by the $1 / f^{3}$ phase noise. The CDC scheme achieves a $6 \mathrm{~dB}$ noise floor suppression compared with the normal differential scheme. This value is smaller than the theoretically predicted $9.6 \mathrm{~dB}$, which is mainly because part of the total $1 / f^{3}$ phase noise is uncorrelated noise from the switch transistors. The frequency counting results for different schemes are shown in Fig.11.

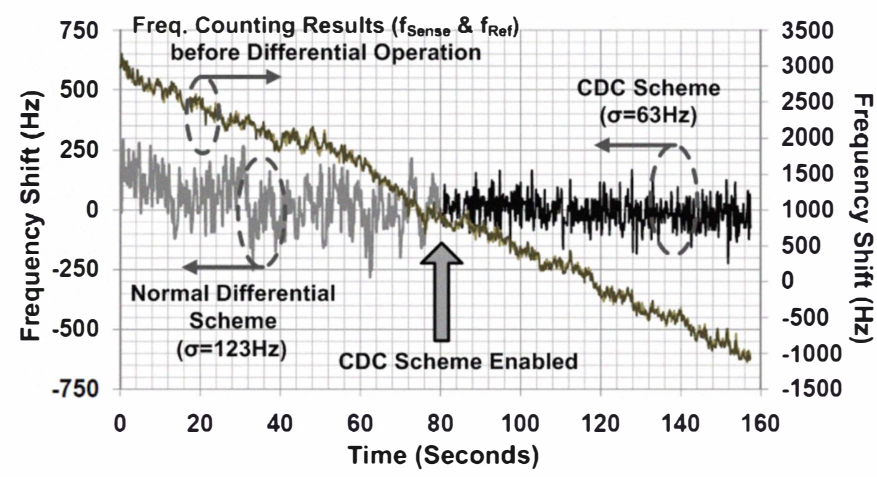

Fig.11. Frequency counting results in time domain for CDC scheme, normal differential and no differential operation.

\section{Sensor Magnetic Sensing Performance}

To verify the magnetic sensing capability of the sensor, DynaBeads ${ }^{\circledR}$ MyOneTM Carboxylic Acid (Diameter $=1 \mu \mathrm{m}$ ), are used as test samples. Solutions with different bead concentrations are deposited onto the sensing site, and the corresponding frequency shifts are measured. The exact quantity of the beads on each site is obtained from scanning electron microscope (SEM) images (Fig.12).

The normalized sensor frequency shift with respect to different bead numbers is shown in Fig.13. The extrapolated frequency shift for one single bead is $342.4 \mathrm{~Hz}$ with an SNR of $14.6 \mathrm{~dB}$ before averaging. The measured sensor response (up to 983 beads) indicates an effective dynamic range of at least 74.5dB. Experiments with bio-samples on genomics level (DNA/RNA) and cellular level (bacteria) are currently under testing. The results will be reported in the near future.

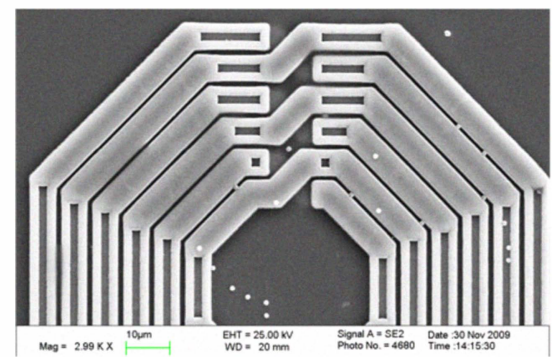

Fig.12. SEM image for one sensor site and deposited magnetic beads.

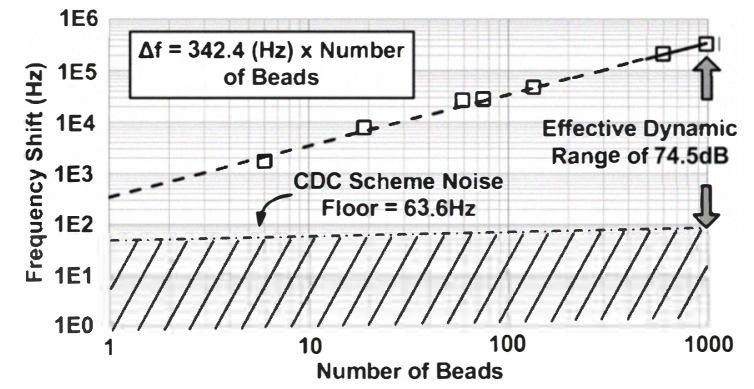

Fig.13. Normalized response for different particle numbers.

\section{CONCLUSION}

In this paper, a scalable ultrasensitive CMOS magnetic sensor array is presented. A noise suppression technique is proposed which increases the sensitivity without any power overhead. A 64-cell sensor array is implemented in a $65 \mathrm{~nm}$ CMOS process, whose measurements verify both the noise suppression capability and the magnetic sensing functionality.

\section{ACKNOWLEDGEMENT}

The authors would like to thank $\mathrm{C}-\mathrm{H}$ Lin and $\mathrm{Z}$. Li of the California Institute of Technology for their technical support on SEM imaging.

\section{REFERENCES}

[1] S. Han, et al., "A High-Density Magnetoresistive Biosensor Array with Drift-Compensation Mechanism," IEEE ISSCC Dig. Tech. Papers, pp.168-169, Feb. 2007.

[2] Y. Liu, et al., "CMOS Mini Nuclear Magnetic Resonance Systemand its Application for Biomolecular Sensing," IEEE ISSCC Dig. Tech. Papers, pp.140-141, Feb. 2008.

[3] P. Besse, et al., "Detection of A Single Magnetic Microbead Using a Miniaturized Silicon Hall Sensor," Appl. Phys.Letters, Vol 80, Issue 22, pp. 4199-4201, Jun. 2002.

[4] H. Wang, et al., "A Frequency-Shift CMOS Magnetic Biosensor Array with Single-Bead Sensitivity and No External Magnet," IEEE ISSCC Dig. Tech. Papers,pp.438-439, Feb. 2009.

[5] A. Hajimiri, et al, "Jitter and Phase Noise in Ring Oscillators," IEEE J. of Solid-State Circuits, vol. 34, no. 6, pp. 790-804, Jun. 1999.

[6] O. Akar, et al, "A Wireless Batch Sealed Absolute Capacitive Pressure Sensor," Sensors and Actuators A: Physcical, vol. 95, no.1, pp. 29-38, Dec. 2001.

[7] C. Liu, et al, "Jitter in Oscillators with $1 / \mathrm{f}$ Noise Sources," ISCAS, pp. 773-776, 2004. 